\title{
Primary malignant melanoma of esophagus: clinicopathologic characterization of 20 cases including molecular genetic profiling of 15 tumors
}

\author{
Jerzy Lasota ${ }^{1}$ - Artur Kowalik $\mathbb{1}^{2} \cdot$ Anna Felisiak-Golabek $^{1} \cdot$ Sebastian Zięba ${ }^{2} \cdot$ Piotr Waloszczyk $^{3} \cdot$ Marek Masiuk $^{4}$. \\ Jaroslaw Wejman $^{5} \cdot$ Justyna Szumilo $^{6} \cdot$ Markku Miettinen $^{1}$
}

Received: 9 July 2018 / Revised: 1 October 2018 / Accepted: 1 October 2018 / Published online: 13 February 2019

(c) United States \& Canadian Academy of Pathology 2018

\begin{abstract}
Primary malignant melanoma of esophagus is very rare, and its clinicopathologic and genetic features have not been extensively investigated. In this study, 20 tumors from 14 male and 6 female patients (40-79 years old) were evaluated. Dysphagia, chest pain, and weight loss were frequent symptoms. Thirteen melanomas, including two with multiple lesions, involved the distal third of esophagus. The median tumor diameter was $6 \mathrm{~cm}$. Epithelioid morphology, moderate atypia, and pigmentation were typical findings. None of the patients had melanoma elsewhere, and all tumors exhibited a junctional periepithelial component consistent with a primary lesion. The median mitotic activity was 11 per 10 high-power fields (range, 0-31). Nine patients died of tumor within 4-22 months, however, two showed long-term (96 and 104 months) survival. In 15 cases, tissue for further immunohistochemical and molecular studies were available. BRAF, KIT, and NRAS mutation status was assessed by Sanger sequencing in all 15 tumors. The next-generation sequencing of 50 or 409 genes was performed in five and three cases, respectively. IGF1R expression indicating activation of the IGF axis was seen in $82 \%$ (9/11) of tumors. However, no BRAF mutations were identified. In 33\% (5/15) of tumors, NRAS mutations were detected. KIT expression was seen in 50\% (7/14) of melanomas including single KIT mutant. Two of three tumors evaluated with 409 genes panel revealed multiple driver mutations indicating sub-clonal expansion, whereas a single mutation (TSC1 p.H371Q) was the sole change in the third case. SF3B1 p.K666T and p.R625C mutations were detected in two cases. However, no cooccurrence of SF3B1 and GNAQ or GNA11 mutations, seen in uveal melanoma, was detected. FBXW7 p.R465C and p. R479G mutations, linked to cancer progression, were found in two of eight tumors. In summary, esophageal melanoma mutation profile indicates complexity of molecular mechanisms underlying its pathogenesis.
\end{abstract}

Electronic supplementary material The online version of this article (https://doi.org/10.1038/s41379-018-0163-y) contains supplementary material, which is available to authorized users.

Jerzy Lasota

jerzy.lasota@nih.gov

1 Laboratory of Pathology, National Cancer Institute, National Institute of Health, Bethesda, MD, USA

2 Department of Molecular Diagnostics, Holycross Cancer Center, Kielce, Poland

3 Zdunomed Independent Laboratory of Pathology, Szczecin, Poland

4 Department of Pathology, Pomeranian Medical University, Szczecin, Poland

5 Center for Medical Postgraduate Education, Warszawa, Poland

6 Department of Clinical Pathomorphology, Medical University of Lublin, Lublin, Poland

\section{Introduction}

Primary malignant melanoma of esophagus (from here on called "esophageal melanoma") is an extremely rare neoplasm with the incidence estimated to be 0.03 per million in the USA [1]. Because of its rarity, only a few small series of esophageal melanoma are available [2-6] and most clinicopathologic data are derived from $>300$ single case studies published since the first histologic description [7-9].

Esophageal melanoma is believed to develop from melanocytes anchored in the esophageal mucosa. Aberrant migration of melanoblasts to the esophagus can occur during their early migration from the neural crest to the epidermis and other sites [10-12]. Primary esophageal melanoma should not be confused with metastatic melanoma, which could present in any portion of the gastrointestinal tract. Although involvement of esophagus is 
uncommon, the differential diagnosis between metastatic and primary melanoma can be challenging [5, 13, 14]. Metastatic melanoma cells can infiltrate mucosa mimicking primary junctional changes [15]. Thus, a primary mucosal melanoma should be defined by identification of melanocytes at the epithelial-stromal junction and/or an adjacent melanoma in situ, and lack of primary cutaneous melanoma [16].

The mutation profile of esophageal melanoma remains incompletely characterized. Sanger sequencing data available of 30 cases are limited to BRAF, NRAS, and KIT mutation status [17-20]. More recent studies have employed next-generation sequencing for multiple targets, or whole-genome sequencing, on a few primary and metastatic tumors [21-24]. However, those studies did not clearly state the diagnostic criteria for primary esophageal melanoma so that metastatic melanomas might have been included.

The aim of this study was to evaluate the clinicopathologic and molecular genetic profile of 20 strictly defined primary esophageal melanomas containing junctional melanocytes.

\section{Materials and methods}

This study evaluated a series of 20 primary malignant melanomas of esophagus. Sixteen tumors were from the Armed Forces Institute of Pathology, Washington, D.C. Additional four tumors were contributed by co-authors. Demographic, clinical, and follow-up data were obtained according to the Institutional Review Board approvals.

\section{Immunohistochemical studies}

Expression of several antigens including melanocytic differentiation markers (human melanoma black $[\mathrm{HMB}]-45$, KBA.62-melanoma associated antigen, Melan-A protein, microphthalmia-associated transcription factor [MITF], PNL-2-melanoma associated antigen, S100 protein, tyrosinase [TYR]) and CD34, CD117 (KIT), cytokeratin 8 (CK8), Cytokeratin cocktail (AE1/AE3), DOG1 (discovered on GIST1; also known as anoctamin 1, or ANO1), insulinlike growth factor 1 receptor (IGF1R), and Vimentin was evaluated immunohistochemically. Leica Bond-Max automated immunostainer (Leica, Bannockburn, IL) was used in this study. Detailed description of antibodies and immunohistochemical protocols is provided in supplemental data.

\section{Molecular genetic studies}

In 15 cases, formalin-fixed paraffin-embedded tissue blocks or unstained tumor sections were available. DNA was extracted from 5 to $105 \mu$ sections using Maxwell ${ }^{\circledR}$ RSC DNA FFPE kit and Maxwell ${ }^{\circledR}$ RSC instrument (Promega, Madison, WI) following manufacturer's protocol provided at www.promega.com. All 15 tumors were screened for $B R A F, K I T$, and NRAS mutations by PCR amplification and Sanger sequencing following previously published protocols [25, 26]. Subsequently, eight tumors with betterpreserved DNA (cases 1, 6, 7, 8, 9, 12, 14, and 20) were evaluated by targeted next-generation sequencing. Ion Torrent $^{\mathrm{TM}}$ (Life Technologies/Thermo Fisher Scientific, Waltham, MA) next-generation sequencing platform was used following manufacturer's recommendations. Depending on the DNA quality either Ion AmpliSeq ${ }^{\mathrm{TM}}$ Cancer Hotspot Panel v2 Kit (50 gene targets) or Ion AmpliSeq ${ }^{\mathrm{TM}}$ Comprehensive Cancer Panel (409 gene targets) was employed to evaluate five and three tumors, respectively. Fifty genes targeted by the Cancer Hotspot Panel were included in the Comprehensive Cancer Panel. A list of all genes analyzed in this study is provided in supplemental data.

The data were processed by Torrent Server Suite 4.2 and sequences aligned to human genome reference sequence HG-19 (The Genome Reference Consortium). Variant calling was performed using Variant Caller v4.2, which is compatible with the Integrative Genomics Viewer (Broad Institute, Cambridge, MA), a high-performance visualization tool for interactive exploration of large, integrated data sets. Mutation nomenclature is based on Human Genome Mutation Society (www.hgvs.org) recommendations. The FATHMM (Functional Analysis Through Hidden Markov Models) scores predicting functional consequences of coding variants were obtained from the COSMIC (Catalog of Somatic Mutations in Cancer) at https://cancer.sanger.ac.uk or assessed using VarSome (The Human Genomic Variant Search Engine) at https://varsome.com.

\section{Results}

\section{Demographic and clinicopathologic data}

There were 14 males and 6 females (ratio 2.3:1). The median age at the diagnosis was 60 years for men and 63.5 years for women. Caucasian ethnicity was known in 16 cases. Demographic and clinicopathologic data are summarized in Table 1. Clinicopathologic characteristics of cases 5 and 8 were previously published [27, 28]. The latter was metachronous melanoma diagnosed 67 months after successful treatment of primary gastric melanoma located in the cardia [28]. Symptoms preceding the diagnosis most commonly included progressive dysphagia $(87 \%$ of the cases), abdominal or chest pain $(40 \%)$, and substantial weight loss (20\%). Distal third of the esophagus was the 
Table 1 Demographic and clinicopathologic data of 20 primary esophageal melanomas evaluated in this study

\begin{tabular}{|c|c|c|c|c|c|c|c|c|c|}
\hline \multirow[t]{2}{*}{ Case } & \multirow[t]{2}{*}{ Sex } & \multirow[t]{2}{*}{ Age } & \multicolumn{3}{|l|}{ Symptoms } & \multirow[t]{2}{*}{ Tumor location } & \multirow[t]{2}{*}{ Tumor size $(\mathrm{cm})$} & \multirow{2}{*}{$\begin{array}{l}\text { Local metastases at the } \\
\text { surgery }\end{array}$} & \multirow{2}{*}{$\begin{array}{l}\text { Follow-up (in } \\
\text { months) }\end{array}$} \\
\hline & & & Dysphagia & Pain & Weight loss & & & & \\
\hline 1 & M & 40 & Unknown & Unknown & Unknown & Distal & $7 \times 3 \times 3$ & No & Alive (96) \\
\hline 2 & M & 45 & Yes & No & No & Distal & 5.5 & Yes & DOD (19) \\
\hline 3 & M & 47 & Yes & No & No & Mid & Unknown & Unknown & DUNK (18) \\
\hline 4 & M & 51 & Yes & Yes & No & Distal & $6 \times 3 \times 5$ & Yes & DOD (10) \\
\hline $5 *$ & M & 57 & Yes & Yes & Yes & Mid & 7 & No & DOPC \\
\hline 6 & M & 58 & Unknown & Unknown & Unknown & Unknown & $3 \times 2.5$ & Yes & DOD (20) \\
\hline 7 & M & 59 & Yes & No & No & Mid to distal & $12.5 \times 4$ & Yes & DOD (11) \\
\hline $8 *$ & M & 61 & Yes & No & No & Mid to distal & 0.7 & No & DOD (16) \\
\hline 9 & M & 62 & No & yes & No & $\begin{array}{l}\text { Distal at E_G } \\
\text { junction }\end{array}$ & $5 \times 4$ & Unknown & Unknown \\
\hline 10 & M & 65 & No & Yes & No & Unknown & $6 \times 6 \times 3$ & No & DOD (4) \\
\hline 11 & M & 68 & Yes & Yes & Yes & Distal & $6 \times 3.5$ & Yes & DOD (22) \\
\hline 12 & M & 72 & Unknown & Unknown & Unknown & $\begin{array}{l}\text { Distal at E_G } \\
\text { junction }\end{array}$ & $\begin{array}{l}2.5 \times 2.3 \times 1+\text { two more } \\
\text { lesions }\end{array}$ & No & DUNK (7) \\
\hline 13 & M & 73 & Yes & No & No & $\begin{array}{l}\text { Distal at E_G } \\
\text { junction }\end{array}$ & 8 & Yes & Unknown \\
\hline 14 & M & 79 & Yes & Yes & Yes & Distal & $7.5 \times 4.5$ & Yes & DOPC \\
\hline 15 & $\mathrm{~F}$ & 53 & Yes & No & No & Distal & $8 \times 1.5$ & Yes & DOD (16) \\
\hline 16 & $\mathrm{~F}$ & 55 & Yes & No & No & Distal & $4 \times 1$ & Yes & Unknown \\
\hline 17 & $\mathrm{~F}$ & 58 & Yes & No & No & $\begin{array}{l}\text { Distal at E_G } \\
\text { junction }\end{array}$ & $12 \times 12 \times 7.5$ & Yes & DOD (4) \\
\hline 18 & $\mathrm{~F}$ & 69 & Unknown & Unknown & Unknown & Distal & $\begin{array}{l}\text { Two lesions: } 3.5 \times 2.5 \times \\
1.5 ; 3 \times 2 \times 1\end{array}$ & No & DUNK (104) \\
\hline 19 & $\mathrm{~F}$ & 71 & Unknown & Unknown & Unknown & Unknown & $2.1 \times 1.3 \times 0.7$ & No & DUNK (8) \\
\hline 20 & $\mathrm{~F}$ & 78 & Yes & No & No & $\begin{array}{l}\text { Distal at E_G } \\
\text { junction }\end{array}$ & $7 \times 4.5 \times 4.5$ & No & Unknown \\
\hline
\end{tabular}

$D O D$ died of disease, $D O P C$ died of postoperative complications, $D U N K$ died of unknown causes, $E_{-} G$ esophagogastric, $L T F$ lost to follow-up, * some data previously reported $[27,28]$

most common location (13 cases), with five of these tumors seated at the esophagogastric junction. The tumor extended to or was limited to the mid-esophagus in four cases. The location was not specified in the remaining three cases. Two patients had multiple lesions. Most esophageal melanomas formed polypoid and lobulated endophytic masses. Tumor size, available in 19 cases, varied from 0.7 to $12 \mathrm{~cm}$ (median $6 \mathrm{~cm}$ ). Ulceration was seen in 17 of 19 cases with suitable data. Metastases in local lymph nodes were detected at the time of surgery in 50\% (9/18) of patients. Yet, in all cases, there was no evidence of co-existing or previous cutaneous melanoma.

\section{Histological features}

A junctional peri-epithelial tumor component was present in all cases and at least focal melanin pigmentation in $85 \%$ $(17 / 20)$ of cases. Pagetoid involvement of the overlying squamous epithelium was seen in $32 \%(6 / 19)$ of melanomas. Majority of tumors were composed of epithelioid cells.
One tumor showed predominantly spindle cell morphology, and focal spindle or round cell component was seen in four cases and nuclear pleomorphism in five cases. Mitotic activity per 10 high-power fields (HPFs; $2 \mathrm{~mm}^{2}$ ) varied from 0 to 31 (median 12). Tumor necrosis was seen in $21 \%$ (4/19) and ulceration in 90\% (17/19) of cases, respectively. Histopathologic data are summarized in Table 2. Representative histological images are shown in Figs. 1a, 2a. Additional figure illustrating junctional changes (atypical melanocytes disposed as single cells and as irregular nests along the basal layer of the esophageal epithelium) in case 19 is available in supplemental data.

\section{Immunohistochemical features}

All analyzed tumors were positive for at least for one marker of melanocytic differentiation (Fig. 2b). IGF1R expression was seen in $83 \%(9 / 11)$ evaluated tumors. Although IGF1R expression pattern was diffuse in all cases (Fig. 1b), intensity of immunohistochemical reactions 
Table 2 Histopathologic features of 20 primary esophageal melanomas

\begin{tabular}{|c|c|c|c|c|c|c|c|c|}
\hline Case & Cell type & Pigment & Atypia & $\begin{array}{l}\text { Mitoses/10 } \\
\text { HPF }\end{array}$ & $\begin{array}{l}\text { Tumor } \\
\text { necrosis }\end{array}$ & $\begin{array}{l}\text { Junctional } \\
\text { activity }\end{array}$ & $\begin{array}{l}\text { Pagetoid } \\
\text { spread }\end{array}$ & Ulceration \\
\hline 1 & Epithelioid to spindle & No & Moderate & 11 & Yes & Yes & No & Yes \\
\hline 2 & Epithelioid & No & Moderate & 10 & No & Yes & No & Yes \\
\hline 3 & Epithelioid & Yes & Moderate & 0 & No & Yes & No & Yes \\
\hline 4 & Epithelioid & Yes & $\begin{array}{l}\text { Moderate (focally } \\
\text { severe) }\end{array}$ & 15 & No & Yes & No & Yes \\
\hline $5^{*}$ & Epithelioid & Yes & Moderate & 6 & No & Yes & No & Yes \\
\hline 6 & Epithelioid & Yes & Moderate & 6 & No & Yes & Yes (f) & Yes \\
\hline 7 & Epithelioid & Yes & $\begin{array}{l}\text { Moderate (focally } \\
\text { severe) }\end{array}$ & 14 & Yes & Yes & No & Yes \\
\hline $8^{*}$ & Epithelioid & Yes & Moderate & 4 & No & Yes & No & No \\
\hline 9 & Epithelioid & Yes & Moderate & 4 & No & Yes & No & Yes \\
\hline 10 & Epithelioid & Yes & Moderate & 23 & No & Yes & Yes & Yes \\
\hline $11^{\S}$ & Epithelioid & Yes & Moderate & $\mathrm{n} / \mathrm{a}$ & $\mathrm{n} / \mathrm{a}$ & Yes & $\mathrm{n} / \mathrm{a}$ & $\mathrm{n} / \mathrm{a}$ \\
\hline 12 & $\begin{array}{l}\text { Epithelioid to round } \\
\text { cell }\end{array}$ & Yes & Moderate & 31 & No & Yes & Yes & Yes \\
\hline 13 & Epithelioid to spindle & Yes & Moderate & 19 & Yes & Yes & No & Yes \\
\hline 14 & Epithelioid & Yes & $\begin{array}{l}\text { Moderate (focally } \\
\text { severe) }\end{array}$ & 9 & No & Yes & No & Yes \\
\hline 15 & Epithelioid & Yes & Moderate & 2 & No & Yes & No & Yes \\
\hline 16 & Epithelioid & Yes & Moderate & 11 & Yes & Yes & Yes & Yes \\
\hline 17 & Spindle to epithelioid & Yes & Severe & 14 & No & Yes & No & Yes \\
\hline 18 & Epithelioid & No & $\begin{array}{l}\text { Moderate (focally } \\
\text { severe) }\end{array}$ & 7 & No & Yes & Yes (f) & Yes \\
\hline 19 & Epithelioid & Yes & Moderate & 12 & No & Yes & Yes (f) & No \\
\hline 20 & Epithelioid & Yes & Moderate & 29 & No & Yes & No & Yes \\
\hline
\end{tabular}

$H P F$ high-power fields, $f$ focal, $n / a=$ not available, $\S$ diagnostic biopsy, * some data previously reported [27, 28]

varied from weak $(n=2)$ to moderate $(n=4)$ and strong $(n$ $=3)$. Fifty percent $(7 / 14)$ of esophageal melanomas showed variable KIT expression (Figs. 1c, d) with diffuse, strong immunoreactivity seen in two cases. In general, KIT expression was more prominent in junctional areas. No CD34 or DOG1 expression was detected. One tumor, case 20, revealed cytokeratin immunoreactivity (focal with CK8 antibody and more prominent with AE1/AE3 cytokeratin cocktail antibody). Vimentin was expressed in $89 \%$ (8/9) of melanomas. Representative images are shown in Figs. 2c, d. Immunohistochemical results are detailed in Table 3.

\section{Molecular genetic features}

Thirty-three percent $(5 / 15)$ of tumors harbored NRAS mutations. A $\mathrm{Q}$ to $\mathrm{K}(n=3)$ and $\mathrm{Q}$ to $\mathrm{H}(n=1)$ substitutions at NRAS codon 61 were the most common change. In one case, p.A146T mutations were identified. One tumor contained a KRAS codon 13 mutation at relatively (20\%) low frequency. Also, in one case, activating KIT mutation (p.L576P) was identified.

Two of three cases studied using Ion AmpliSeq ${ }^{\mathrm{TM}}$ Comprehensive Cancer Panel next-generation sequencing contained SF3B1 (splicing factor 3B subunit 1) mutations (p.R625C and p.K666T). A p.R625C co-occurred with nonsense mutation truncating mammalian target of rapamycin (mTOR) at p.Q1684, whereas p.K666T was detected in melanoma carrying NRAS p.Q61K and KIT p.L576P driver mutations in addition to cyclin-dependent kinase inhibitor 2B (CDKN2B) p.110N and ataxia telangiectasia and $\operatorname{Rad} 3$ related (ATR) p.V372G substitutions. In one case, tuberous sclerosis 1 (TSC1) p.H371Q was the only mutation identified. Two F-box and WD repeat domain containing 7 (FBXW7) mutants, p.R465C and p.R479G were found among eight analyzed cases. Tumor characterized by FBXW7 p.R465C also harbored KRAS p.G13C mutations at a low frequency. FATHMM scores describing pathogenicity of the missense variants identified in this study ranged from 0.80 to 0.99 supporting pathogenic potential (complete list provided in supplemental data).

In case 20, NRAS p.Q61K and KIT WT genotype, and NRAS WT and KIT p.L576P genotype were identified by Sanger sequencing and next-generation sequencing, respectively. This variation could be related to two different DNA samples evaluated by Sanger and next-generation sequencing. 
Fig. 1 Examples of histologic and immunohistochemical findings in esophageal melanoma. Junctional changes (a) and prominent IGF1R expression (b) in case 6. Strong KIT expression in $K I T$-wild type case 7 (c) and weak KIT expression in KIT mutant case $20(\mathbf{d})$
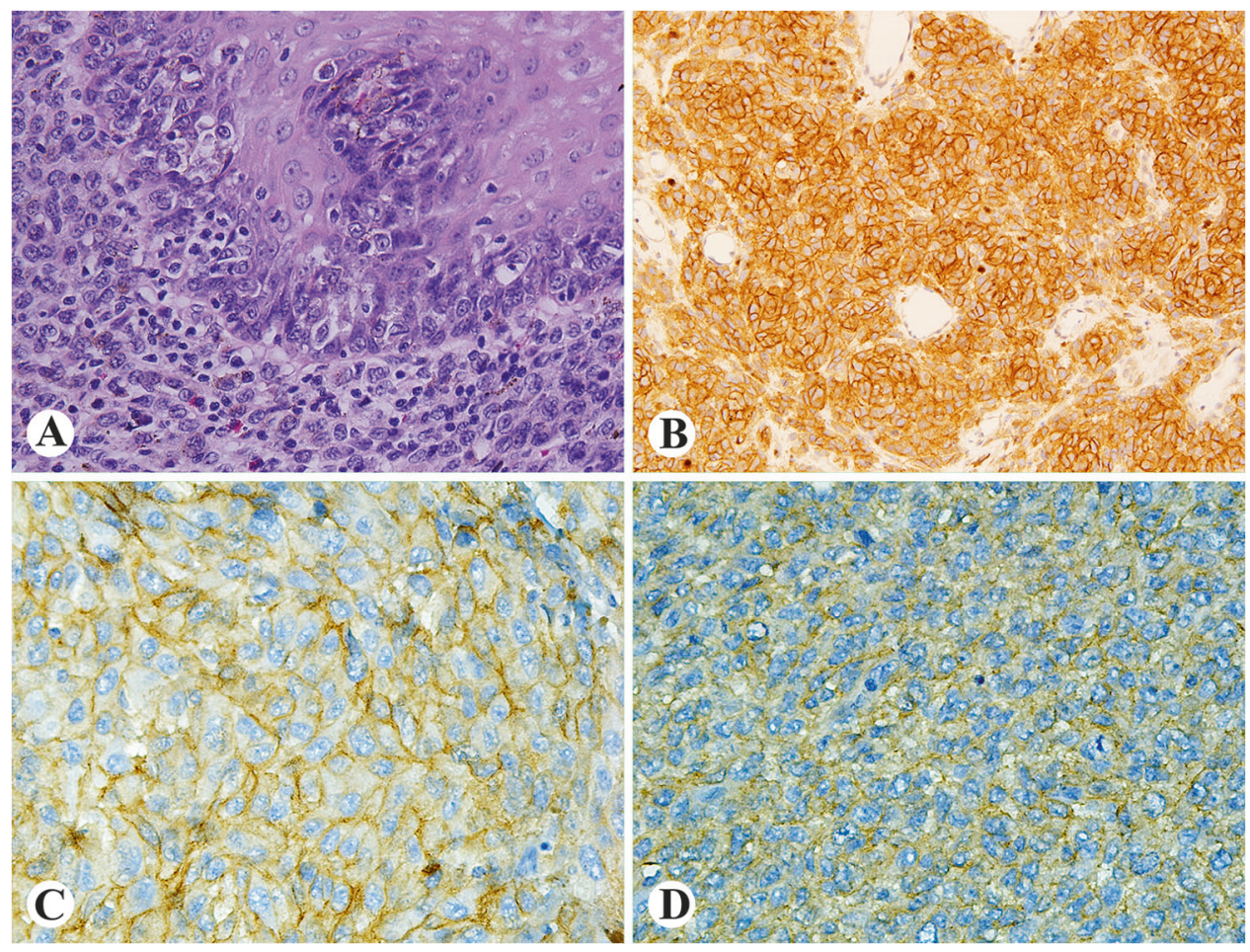

No mutations were identified in the genes often indicated in melanoma [29, 30] such as $B R A F$ (15 cases analyzed), CDKN2A, GAN11 and GNAQ, PIK3CA and TP53 (eight cases analyzed) $B A P 1$ and $N F 1$ (three cases analyzed). Sanger and next-generation sequencing results are detailed in Table 4 and supplemental data.

\section{Follow-up data}

Metastatic disease at the surgery was diagnosed in 9 of 18 (50\%) cases. Follow-up data were available on 16 patients. Two patients including one with local metastases died of postoperative complications. Nine patients died of disease within 4-22 months (mean survival 15 months), while 4 patients died of unknown causes within 8-104 months (mean survival 43 months). One patient was alive without disease 96 months after surgery; two patients, who survived 96 and 104 months, respectively, had no nodal metastases at surgery.

\section{Discussion}

This study analyzed 20 well-documented primary esophageal melanomas. Seventy percent of patients were male. Similar age distribution and predominance of male gender were reported in two recently published largest cohorts of 13 and 17 primary esophageal melanoma patients of Asian ethnicity [31, 32]. Male predominance among primary esophageal melanoma patients is reportedly not associated with alcohol and tobacco consumption, as seen in esophageal squamous cell carcinoma patients [32]. In this series, most of melanomas arose in the distal third of the esophagus, a location frequently indicated by previous studies [9]. Progressive dysphagia accompanied by upper abdominal pain and weight loss mirror reported main clinical symptoms [33].

Tumor ulceration was seen in almost all (17/19) analyzed esophageal melanomas. Presence of ulceration is a prognostic factor indicating shorter overall-survival for both Stage I and Stage II cutaneous melanoma patients [34]. In this study, one of two cases with long overall survival lacked ulceration. Also, mitotic rate has been considered to be a prognostic factor for cutaneous melanoma [35]. In this study, mitotic rates were slightly higher in esophageal melanomas with overall survival shorter than 12 months.

In general, the prognosis for esophageal melanoma is poor $[8,33]$. A great majority of patients included in this series died of disease within several months. However, two long survivals of 104 and 96 months were documented. In both cases, no local lymph node metastases were diagnosed at the curative resection. Patients with esophageal melanoma at T1a stage revealed excellent prognosis compared with more advanced tumors with local lymph nodes metastases [36]. However, long-term survivals (up to 12 years) have been reported in few cases with submucosal invasion and local lymph node metastases treated by subtotal esophagectomy and adjuvant chemotherapy $[37,38]$. 
Fig. 2 Histologic and immunohistochemical findings in case 10. Epithelioid melanoma with moderate atypia and prominent pigmentation (a) strong HMB45 expression (b), showing cytokeratin $(\mathbf{c})$ and vimentin immunoreactivity $(\mathbf{d})$

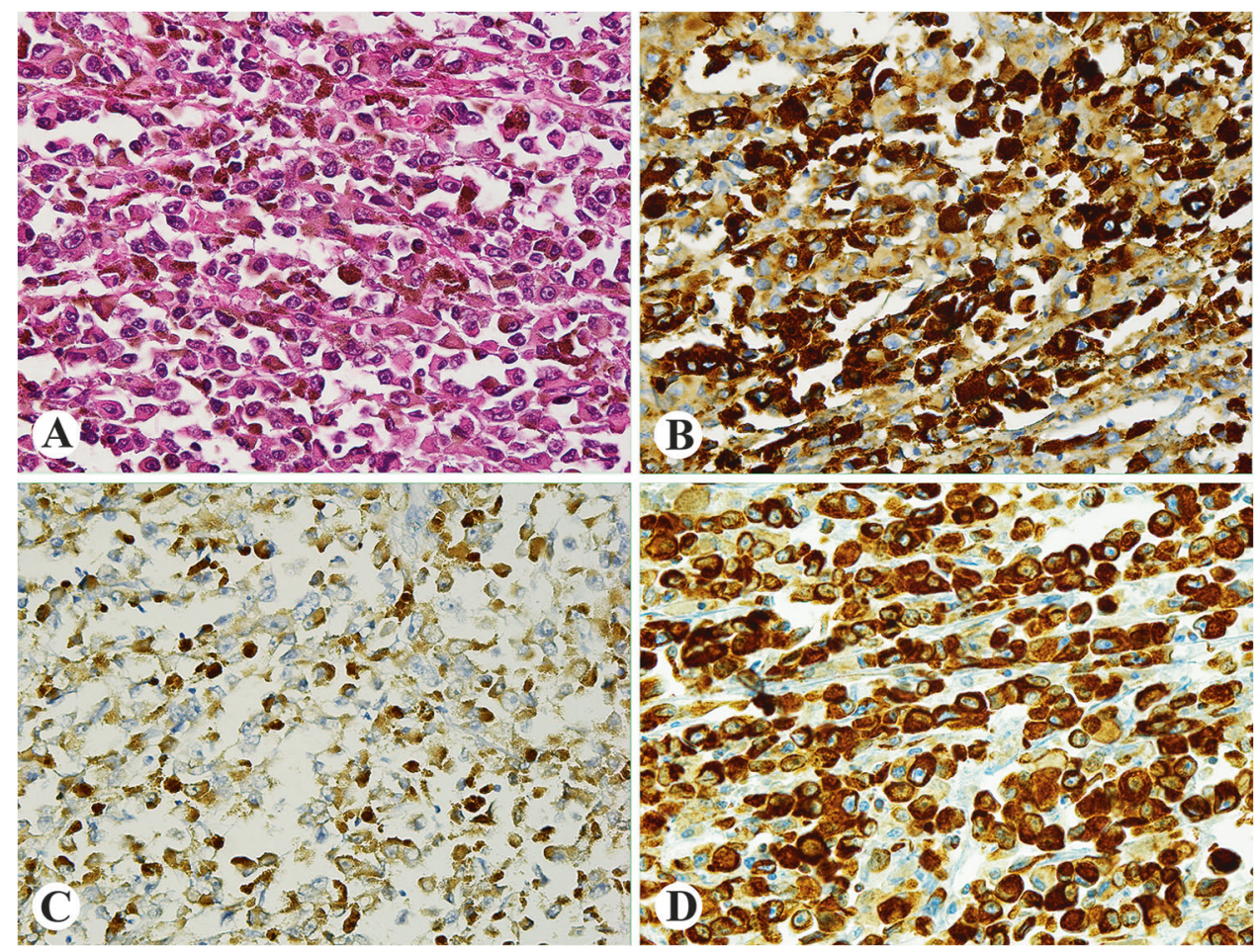

In this series of esophageal melanomas, one tumor expressed keratins. This phenomenon has been previously described [39]. The tumor invasiveness and metastatic potential may correlate with keratin and vimentin coexpression [40]. Reported in this study, tumor coexpressing keratin and vimentin showed rapid progression and only 4-month overall survival.

Alterations of proteins forming MAPK (mitogen-activated protein kinase) pathway that communicates signals from cell surface to the nucleus, have been reported in different type of cancers including malignant melanoma [41]. NRAS (neuroblastoma RAS viral [v-ras] oncogene homolog) is a member of human RAS proto-oncogene family that encodes cell membrane-associated proteins involved in transduction of extracellular growth and differentiation signals [42]. Typically, oncogenic NRAS mutations cluster in exon 1 (G12/13) and exon 2 (Q61) and represent the second most common driver after $B R A F$ mutations in melanoma [43]. About 5-20\% of mucosal melanoma, depending on tumor location, harbor NRAS mutations [44]. In this study, five NRAS mutants were identified among 15 esophageal melanomas. Four substitutions were found in codon 61, a "hot-spot" for NRAS mutations in melanoma [45], whereas one tumor harbored NRAS p.A146T substitution. This mutation has not been reported in melanoma by COSMIC. However, it was identified in melanoma cell line A375 clones with acquired resistance to the dabrafenib GSK2118436, a BRAF inhibitor [46]. NRAS p.A146T mutation was detected in blastic plasmacytoid dendritic cell neoplasm, and in B- and T-cell acute lymphoblastic leukemia [47-49].

KIT, a transmembrane receptor tyrosine kinase, plays a crucial role in growth regulation, differentiation, migration, and proliferation of melanocytes. Somatic KIT mutations cause oncogenic signaling affecting both the mitogenactivated protein kinase (MAPK) and phosphatidylinositol 3-kinase (PI3K) pathway [50]. KIT-mutants were mainly identified among mucosal, acral, and chronically sundamaged skin tumors $[51,52]$. In melanoma, $70 \%$ of KIT mutations were found in juxtamembrane domain (exon 11) with p.L576P substitution being a most common [53]. This mutation, initially reported in gastrointestinal stromal tumors, leads to pathologic activation of KIT tyrosine kinase activity [54]. In this study, p.L576P mutation was found in $7 \%$ (1/15) of esophageal melanomas. Previously, a few $(n=$ 4) KIT mutations including p.L576P, p.H580_G592dup in the juxtamembrane domain, and p.F504L and p. A502_Y503insFA in extracellular domain, were reported in a combined cohort of 17 esophageal melanomas [18, 20, 21]. However, variable KIT expression was detected immunohistochemically in a higher number of cases. In this study, $50 \%$ of analyzed tumors revealed KIT positivity. As reported, KIT was stronger expressed in the in situ and junctional component than in the invasive part of the lesion [55]. Although response to tyrosine kinase inhibitors imatinib mesylate and sorafenib in KIT-mutated rectal melanoma have been reported in isolated cases [56, 57], larger studies have failed to confirm convincing therapeutic efficacy [58]. 
Table 3 Immunohistochemical features of 20 primary esophageal melanomas

\begin{tabular}{|c|c|c|c|c|c|c|c|c|c|c|c|c|c|c|}
\hline \multirow[b]{2}{*}{ Case } & \multicolumn{7}{|c|}{ Melanocytic differentiation markers } & \multicolumn{7}{|c|}{ Other markers } \\
\hline & HMB45 & KBA.62 & Melan-A & MITF & PNL-2 & S100 & TYR & CD34 & $\begin{array}{l}\text { CD117 } \\
\text { (KIT) }\end{array}$ & DOG1 & IGFR1 & $\begin{array}{l}\text { Keratin } \\
\text { (CK8) }\end{array}$ & $\begin{array}{l}\text { Keratins } \\
\text { (pan-CK) }\end{array}$ & VIM \\
\hline 1 & $(+)$ & $(+)$ & $(+)$ & ND & $(+)$ & $(+)$ & $(+)$ & $(-)$ & $(+)$ & ND & $(+)$ & ND & $(-)$ & ND \\
\hline 2 & $(+)$ & ND & ND & ND & ND & $(+)$ & ND & ND & ND & ND & ND & ND & ND & ND \\
\hline 3 & $(+)$ & ND & ND & ND & ND & $(+)$ & ND & ND & $(+)$ & ND & ND & ND & ND & ND \\
\hline 4 & ND & ND & ND & ND & ND & $(+)$ & ND & ND & ND & ND & ND & ND & ND & ND \\
\hline 5 & $(+)$ & $(-)$ & $(-)$ & $(-)$ & $(+)$ & $(-)$ & $(+)$ & $(-)$ & $(-)$ & $(-)$ & $(+)$ & $(-)$ & $(-)$ & $(+)$ \\
\hline 6 & $(+)$ & $(+)$ & $(+)$ & $(+)$ & $(+)$ & $(+)$ & $(+)$ & $(-)$ & $(-)$ & $(-)$ & $(+)$ & $(-)$ & $(-)$ & $(+)$ \\
\hline 7 & $(+)$ & $(-)$ & $(+)$ & $(-)$ & $(+)$ & $(+)$ & $(+)$ & $(-)$ & $(+)$ & $(-)$ & $(-)$ & $(-)$ & $(-)$ & $(+)$ \\
\hline 8 & $(+)$ & $(-)$ & $(+)$ & $(+)$ & $(+)$ & $(+)$ & $(+)$ & $(-)$ & $(-)$ & $(-)$ & $(+)$ & $(-)$ & $(-)$ & $(+)$ \\
\hline 9 & $(-)$ & $(+)$ & $(+)$ & $(+)$ & $(-)$ & $(+)$ & $(-)$ & $(-)$ & $(-)$ & $(-)$ & $(+)$ & $(-)$ & $(-)$ & $(+)$ \\
\hline 10 & $(+)$ & $(+)$ & $(+)$ & $(-)$ & $(+)$ & $(+)$ & $(+)$ & $(-)$ & $(-)$ & $(-)$ & $(-)$ & $(+)$ & $(+)$ & $(+)$ \\
\hline 11 & $(+)$ & ND & ND & ND & ND & $(+)$ & ND & ND & $(+)$ & ND & ND & ND & ND & ND \\
\hline 12 & $(+)$ & $(+)$ & $(+)$ & $(+)$ & $(+)$ & $(+)$ & $(+)$ & $(-)$ & $(+)$ & $(-)$ & $(+)$ & $(-)$ & $(-)$ & $(+)$ \\
\hline 13 & ND & ND & ND & ND & ND & $(+)$ & ND & ND & ND & ND & ND & ND & ND & ND \\
\hline 14 & $(+)$ & $(-)$ & $(-)$ & $(-)$ & $(+)$ & $(+)$ & $(+)$ & $(-)$ & $(-)$ & $(-)$ & $(+)$ & $(-)$ & $(-)$ & $(-)$ \\
\hline 15 & $(+)$ & ND & ND & ND & ND & $(+)$ & ND & ND & ND & ND & ND & ND & ND & ND \\
\hline 16 & $(+)$ & ND & ND & ND & ND & ND & ND & ND & $(+)$ & ND & ND & ND & ND & ND \\
\hline 17 & $(+)$ & ND & ND & ND & ND & ND & ND & ND & ND & ND & ND & ND & ND & ND \\
\hline 18 & ND & ND & ND & ND & ND & $(+)$ & ND & ND & ND & ND & ND & ND & ND & ND \\
\hline 19 & $(+)$ & $(+)$ & $(+)$ & ND & $(+)$ & $(+)$ & ND & ND & $(-)$ & ND & $(+)$ & $(-)$ & $(-)$ & ND \\
\hline 20 & $(+)$ & $(-)$ & $(+)$ & $(-)$ & $(+)$ & $(+)$ & $(+)$ & $(-)$ & $(+)$ & $(-)$ & $(+)$ & $(-)$ & $(-)$ & $(+)$ \\
\hline
\end{tabular}

$N D$ not done

Two of three esophageal melanomas analyzed with Ion AmpliSeq ${ }^{\mathrm{TM}}$ Comprehensive Cancer Panel revealed mutations in the gene encoding splicing factor $3 \mathrm{~B}$ subunit 1 (SF3B1), a component of the spliceosome. Identical SF3B1 p.R625C and p.K666T mutations were previously reported in uveal melanomas, colorectal and other mucosal melanomas, and have been associated with diverse alternative splicing events $[59,60]$. Approximately $20 \%$ of each uvealharbored $S F 3 B 1$ somatic mutations. In uveal melanoma, the presence of SF3B1 mutations is associated with mutational activation of GNAQ or GNA11 oncogenes [59]. In esophageal melanoma, no co-occurrence of $S F 3 B 1$ and $G N A Q$ or GNA11 mutations was identified. However, one SF3B1mutant harbored KIT p.L576P and NRAS p.Q61K mutations. A recent study documented SF3B1 mutations in anorectal melanomas harboring $R A S$ mutations [22]. Second of SF3B1-mutant esophageal melanomas harbored mTOR FAT-domain p.Gln1684* mutation. Previously, missense mTOR mutations were identified in mucosal melanoma and linked to a worse prognosis [61]. Although biological significance of $m T O R$ nonsense mutation is unknown, mTOR inactivation may lead to deregulation of mTOR complex 1 and its tumor suppression function [62].

Two esophageal melanomas harbored mutations affecting the FBXW7 gene. FBXW7 (F-Box and WD repeat domain containing 7) encodes a member of the F-box protein family. The F-box proteins constitute one of the four subunits of ubiquitin protein ligase complex called SCFs (SKP1-cullin-F-box), which functions in phosphorylationdependent ubiquitination. A recent melanoma study showed no association between the presence of $F B X W 7$ and $B R A F$ or $R A S$ mutations and designated FBXW7 as a tumorsuppressor gene, a novel driver for a subset of melanomas [63]. In line with this observation, one of the FBXW7mutant esophageal melanomas reported in this study was $B R A F$ and $R A S$ wild type. However, another tumor harbored a p.G13C KRAS-mutant subclone. In general, KRAS-mutants are very rare $(<1 \%)$ in melanoma and have not been reported in esophageal melanoma. However, concomitant of FBXW7 and KRAS mutations have been found in advanced colorectal carcinomas [64]. Two FBXW7 mutations identified in esophageal melanoma (p. R465C and p.R479G), which are considered to inactivate FBXW7 were previously reported in ovarian and head and neck squamous cell carcinomas [64].

In one esophageal melanoma, a p.H371Q mutation in tuberin-binding domain of TSC1 was identified as a sole alteration. Recent study reported TCS1 mutations in a spectrum of mucosal melanomas. In a few TSC1 mutants, including one esophageal melanoma, alteration of TSC1 
Table 4 Results of esophageal melanoma genotyping including Sanger sequencing of BRAF, KIT, and NRAS (15 cases) and all mutations identified by the Ion Torrent ${ }^{\mathrm{TM}}$ next-generation sequencing with Ion AmpliSeq ${ }^{\mathrm{TM}}$ Cancer Hotspot Panel v2 Kit (five cases) and Ion AmpliSeq Comprehensive Cancer Panel (three cases)

\begin{tabular}{|c|c|c|c|c|c|c|c|c|c|c|c|}
\hline \multirow[b]{2}{*}{ Case } & \multirow[b]{2}{*}{ Methods } & \multicolumn{10}{|l|}{ Gene } \\
\hline & & $A T R$ & $B R A F$ & $C D K N 2 B$ & $F B X W 7$ & $K I T$ & $K R A S$ & $N R A S$ & MTOR & $S F 3 B 1$ & TSC1 \\
\hline 1 & $\begin{array}{l}\text { Ion AmpliSeq }{ }^{\mathrm{TM}} \text { CCP Sanger } \\
\text { sequencing }\end{array}$ & WT & WT & WT & WT & WT & WT & WT & WT & WT & p.H371Q \\
\hline 3 & Sanger sequencing & & WT & & & WT & & WT & & & \\
\hline 5 & Sanger sequencing & & WT & & & WT & & WT & & & \\
\hline 6 & $\begin{array}{l}\text { Ion AmpliSeq }{ }^{\mathrm{TM}} \text { CHP Sanger } \\
\text { sequencing }\end{array}$ & NIP & WT & & WT & WT & WT & p.Q61K & NIP & NIP & NIP \\
\hline 7 & $\begin{array}{l}\text { Ion AmpliSeq }{ }^{\mathrm{TM}} \text { CHP Sanger } \\
\text { sequencing }\end{array}$ & NIP & WT & NIP & p.R479G & WT & WT & WT & NIP & NIP & NIP \\
\hline 8 & $\begin{array}{l}\text { Ion AmpliSeq }{ }^{\mathrm{TM}} \text { CHP Sanger } \\
\text { sequencing }\end{array}$ & NIP & WT & NIP & WT & WT & WT & p.A146T & NIP & NIP & NIP \\
\hline 9 & $\begin{array}{l}\text { Ion AmpliSeq }{ }^{\mathrm{TM}} \text { CHP Sanger } \\
\text { sequencing }\end{array}$ & NIP & WT & NIP & WT & WT & WT & p.Q61K & NIP & NIP & NIP \\
\hline 10 & Sanger sequencing & & WT & & & WT & & WT & & & \\
\hline 11 & Sanger sequencing & & WT & & & WT & & WT & & & \\
\hline 12 & $\begin{array}{l}\text { Ion AmpliSeq }{ }^{\mathrm{TM}} \text { CCP Sanger } \\
\text { sequencing }\end{array}$ & WT & WT & WT & WT & WT & WT & WT & p.Q1684* & p.R625C & WT \\
\hline 14 & $\begin{array}{l}\text { Ion AmpliSeq }{ }^{\mathrm{TM}} \text { CHP Sanger } \\
\text { sequencing }\end{array}$ & NIP & WT & NIP & p.R465C & WT & p.G13C & WT & NIP & NIP & NIP \\
\hline 16 & Sanger sequencing & & WT & & & WT & & p.Q61H & & & \\
\hline 17 & Sanger sequencing & & WT & & & WT & & WT & & & \\
\hline 19 & Sanger sequencing & & WT & & & WT & & WT & & & \\
\hline 20 & $\begin{array}{l}\text { Ion AmpliSeq }{ }^{\mathrm{TM}} \text { CCP Sanger } \\
\text { sequencing }\end{array}$ & p.V372G & WT & p.D110N & WT & ${ }^{\mathrm{x}}$ p.L576P & WT & ${ }^{\mathrm{xx}} \mathrm{p} \cdot \mathrm{Q} 61 \mathrm{~K}$ & WT & p.K666T & WT \\
\hline
\end{tabular}

Complete next-generation sequencing data are provided in the Supplemental Table 2 and Supplemental Table 3

CCP Comprehensive Cancer Panel (409 gene targets), CHP Cancer Hotspot Panel v2 Kit (50 gene targets), NIP not included in this panel, ${ }^{\mathrm{x}}$ nextgeneration sequencing result, ${ }^{\mathrm{xx}}$ Sanger sequencing result

was the only change and did not co-occur with NRAS, KIT, or BRAF mutations [65]. In contrast with those cases, tumors with multiple driver mutations were seen in this series and previously reported indicating high frequency of somatic mutation in melanoma [66]. Dynamic clonal changes might be responsible for the differences between Sanger and NGS-sequencing results in case 20, especially if different DNA samples are being evaluated.

In this study, no BRAF mutations were identified in esophageal melanomas. Previous investigations have reported a small number of $B R A F$-mutant esophageal melanomas [18, 20-24, 66, 67]. However, clinical and histological findings specific for primary esophageal melanoma were not clearly documented, so that the possibility of inclusion of metastatic cutaneous melanoma in those series cannot be excluded. In this study, both atypical junctional changes in the squamous epithelium and/or an adjacent melanoma in situ with no evidence for co-existing or previous cutaneous melanoma was documented in all cases.

In summary, activation of RAS_RAF_MEK pathway through the NRAS mutations seems to be essential for development of a subset of esophageal melanoma, whereas $B R A F$ mutations are rare if they occur. Also, mutations of $F B X 7, K I T, S F 3 B 1$, and $T S C 1$ being previously found in other mucosal melanomas may play significant role in this tumor with a complex pathogenesis. Further studies, such as RNA sequencing for fusion gene transcripts, may identify other molecular events underlying initiation and progression of this rare neoplasm.

Acknowledgements We thank Dr. Leslie Sobin, MD, for support and reviewing this manuscript.

\section{Compliance with ethical standards}

Conflict of interest The authors declare that they have no conflict of interest.

\section{References}

1. Coté TR, Sobin LH. Primary melanomas of the esophagus and anorectum: epidemiologic comparison with melanoma of the skin. Melanoma Res. 2009;19:58-60. 
2. DiCostanzo DP, Urmacher C. Primary malignant melanoma of the esophagus. Am J Surg Pathol. 1987;11:46-52.

3. Lohmann CM, Hwu WJ, Iversen K, et al. Primary malignant melanoma of the oesophagus: a clinical and pathological study with emphasis on the immunophenotype of the tumours for melanocyte differentiation markers and cancer/testis antigens. Melanoma Res. 2003;13:595-601.

4. Li B, Lei W, Shao K, et al. Characteristics and prognosis of primary malignant melanoma of the esophagus. Melanoma Res. 2007;17:239-42.

5. Sanchez AA, Wu TT, Prieto VG, et al. Comparison of primary and metastatic malignant melanoma of the esophagus: clinicopathologic review of 10 cases. Arch Pathol Lab Med. 2008;132:1623-9.

6. Yu H, Huang XY, Li Y, et al. Primary malignant melanoma of the esophagus: a study of clinical features, pathology, management and prognosis. Dis Esophagus. 2011;24:109-13.

7. Garfinkle JM, Cahan WG. Primary melanocarcinoma of the esophagus; first histologically proved case. Cancer . 1952;5:921-6.

8. Volpin E, Sauvanet A, Couvelard A, et al. Primary malignant melanoma of the esophagus: a case report and review of the literature. Dis Esophagus. 2002;15:244-9.

9. Bisceglia M, Perri F, Tucci A, et al. Primary malignant melanoma of the esophagus: a clinicopathologic study of a case with comprehensive literature review. Adv Anat Pathol. 2011;18:235-52.

10. De La Pava S, Nigogosyan G, Pickren JW, et al. Melanosis of the esophagus. Cancer . 1963;16:48-50.

11. Tateishi R, Taniguchi H, Wada A, et al. Argyrophil cells and melanocytes in esophageal mucosa. Arch Pathol. 1974;98:87-9.

12. Ohashi K, Kato Y, Kanno J, et al. Melanocytes and melanosis of the oesophagus in Japanese subjects--analysis of factors effecting their increase. Virchows Arch A Pathol Anat Histopathol. 1990;417:137-43.

13. Eng J, Pradhan GN, Sabanathan S, et al. Malignant melanoma metastatic to the esophagus. Ann Thorac Surg. 1989;48:287-88.

14. Schneider A, Martini N, Burt ME. Malignant melanoma metastatic to the esophagus. Ann Thorac Surg. 1993;55:516-7.

15. Littman CD. Metastatic melanoma mimicking primary bronchial melanoma. Histopathology. 1991;18:561-3.

16. Allen AC, Spitz S. Malignant melanoma; a clinicopathological analysis of the criteria for diagnosis and prognosis. Cancer . 1953:6:1-45.

17. Wong CW, Fan YS, Chan TL, et al. Cancer Genome Project. BRAF and NRAS mutations are uncommon in melanomas arising in diverse internal organs. J Clin Pathol. 2005;58:640-4.

18. Sekine S, Nakanishi Y, Ogawa R, et al. Esophageal melanomas harbor frequent NRAS mutations unlike melanomas of other mucosal sites. Virchows Arch. 2009;454:513-7.

19. Terada T. Amelanotic malignant melanoma of the esophagus: report of two cases with immunohistochemical and molecular genetic study of KIT and PDGFRA. World J Gastroenterol. 2009;15:2679-83.

20. Langer R, Becker K, Feith M, et al. Genetic aberrations in primary esophageal melanomas: molecular analysis of c-KIT, PDGFR, KRAS, NRAS and BRAF in a series of 10 cases. Mod Pathol. 2011;24:495-501.

21. Furney SJ, Turajlic S, Stamp G, et al. Genome sequencing of mucosal melanomas reveals that they are driven by distinct mechanisms from cutaneous melanoma. J Pathol. 2013;230:261-9.

22. Cosgarea I, Ugurel S, Sucker A, et al. Targeted next generation sequencing of mucosal melanomas identifies frequent NF1 and RAS mutations. Oncotarget. 2017;8:40683-92.

23. Zehir A, Benayed R, Shah RH, et al. Mutational landscape of metastatic cancer revealed from prospective clinical sequencing of 10,000 patients. Nat Med. 2017;23:703-13.
24. Li J, Yan S, Liu Z, et al. Multiregional sequencing reveals genomic alterations and clonal dynamics in primary malignant melanoma of the esophagus. Cancer Res. 2018;78:338-47.

25. Lasota J, Kowalik A, Wasag B, et al. Detection of the BRAF V600E mutation in colon carcinoma: critical evaluation of the imunohistochemical approach. Am J Surg Pathol. 2014;38:1235-41.

26. Lasota J, Jasinski M, Sarlomo-Rikala M, et al. Mutations in exon 11 of c-Kit occur preferentially in malignant versus benign gastrointestinal stromal tumors and do not occur in leiomyomas or leiomyosarcomas. Am J Pathol. 1999;154:53-60.

27. Niezychowska K, Zawadzki J, Wejman J. Primary malignant melanoma of the esophagus. A case report. Pol J Pathol. 1997;48:205-7.

28. Dabrowski A, Zinkiewicz K, Szumilo J, et al. Unusual clinical course of metachronous melanomas of the upper digestive system. World J Gastroenterol. 2005;11:2197-9.

29. Hayward NK, Wilmott JS, Waddell N, et al. Whole-genome landscapes of major melanoma subtypes. Nature. 2017;545:175-80.

30. Hintzsche JD, Gorden NT, Amato CM, et al. Whole-exome sequencing identifies recurrent SF3B1 R625 mutation and comutation of NF1 and KIT in mucosal melanoma. Melanoma Res. 2017;27:189-99.

31. Wang S, Tachimori Y, Hokamura N, et al. Diagnosis and surgical outcomes for primary malignant melanoma of the esophagus: a single-center experience. Ann Thorac Surg. 2013;96:1002-6.

32. Gao Shugeng, Li Jiagen, Feng Xiaoli, et al. Characteristics and surgical outcomes for primary malignant melanoma of the esophagus. Sci Rep. 2016;6:23804.

33. Sabanathan S, Eng J, Pradhan GN. Primary malignant melanoma of the esophagus. Am J Gastroenterol. 1989;84:1475-81.

34. Balch CM, Wilkerson JA, Murad TM, et al. The prognostic significance of ulceration of cutaneous melanoma. Cancer . 1980;45:3012-7.

35. Attis MG, Vollmer RT. Mitotic rate in melanoma: a reexamination. Am J Clin Pathol. 2007;127:380-4.

36. Kuwabara S, Ebihara Y, Nakanishi Y, et al. Primary malignant melanoma of the esophagus treated with subtotal esophagectomy: a case report. BMC Surg. 2017;17:122.

37. Hamdy FC, Smith JH, Kennedy A, et al. Long survival after excision of a primary malignant melanoma of the oesophagus. Thorax. 1991;46:397-8.

38. Gupta V, Kochhar R, Sinha SK, et al. Primary malignant melanoma of the esophagus: long-term survival after radical resection. J Thorac Oncol. 2009;4:1180-2.

39. Miettinen M, Franssila K. Immunohistochemical spectrum of malignant melanoma. The common presence of keratins. Lab Invest. 1989;61:623-8.

40. Hendrix MJ, Seftor EA, Chu YW, et al. Coexpression of vimentin and keratins by human melanoma tumor cells: correlation with invasive and metastatic potential. $\mathrm{J}$ Natl Cancer Inst. 1992;84:165-74.

41. Davies H, Bignell GR, Cox C, et al. Mutations of the BRAF gene in human cancer. Nature. 2002;417:949-54.

42. Pylayeva-Gupta Y, Grabocka E, Bar-Sagi D. RAS oncogenes: weaving a tumorigenic web. Nat Rev Cancer. 2011;11:761-74.

43. Hawryluk EB, Tsao H. Melanoma: clinical features and genomic insights. Cold Spring Harb Perspect Med. 2014;4:a015388.

44. Tacastacas JD, Bray J, Cohen YK, et al. Update on primary mucosal melanoma. J Am Acad Dermatol. 2014;71:366-75.

45. Jakob JA, Bassett RL Jr, Ng CS, et al. NRAS mutation status is an independent prognostic factor in metastatic melanoma. Cancer . 2012;118:4014-23.

46. Greger JG, Eastman SD, Zhang V, et al. Combinations of BRAF, $\mathrm{MEK}$, and $\mathrm{PI} 3 \mathrm{~K} / \mathrm{mTOR}$ inhibitors overcome acquired resistance 
to the BRAF inhibitor GSK2118436 dabrafenib, mediated by NRAS or MEK mutations. Mol Cancer Ther. 2012;11:909-20.

47. Menezes J, Acquadro F, Wiseman M, et al. Exome sequencing reveals novel and recurrent mutations with clinical impact in blastic plasmacytoid dendritic cell neoplasm. Leukemia. 2014;28:823-9.

48. Neumann M, Vosberg S, Schlee C, et al. Mutational spectrum of adult T-ALL. Oncotarget. 2015;6:2754-66.

49. de Smith AJ, Ojha J, Francis SS, et al. Clonal and microclonal mutational heterogeneity in high hyperdiploid acute lymphoblastic leukemia. Oncotarget. 2016;7:72733-45.

50. Lennartsson J, Jelacic T, Linnekin D, et al. Normal and oncogenic forms of the receptor tyrosine kinase kit. Stem Cells. 2005;23:16-43.

51. Handolias D, Salemi R, Murray W, et al. Mutations in KIT occur at low frequency in melanomas arising from anatomical sites associated with chronic and intermittent sun exposure. Pigment Cell Melanoma Res. 2010;23:210-5.

52. Abu-Abed S, Pennell N, Petrella T, et al. KIT gene mutations and patterns of protein expression in mucosal and acral melanoma. $\mathrm{J}$ Cutan Med Surg. 2012;16:135-42.

53. Woodman SE, Davies MA. Targeting KIT in melanoma: a paradigm of molecular medicine and targeted therapeutics. Biochem Pharmacol. 2010;80:568-74.

54. Shtivelman E, Davies MQ, Hwu P, et al. Pathways and therapeutic targets in melanoma. Oncotarget. 2014;5:1701-52.

55. Montone KT, van Belle P, Elenitsas R, et al. Proto-oncogene c-kit expression in malignant melanoma: protein loss with tumor progression. Mod Pathol. 1997;10:939-44.

56. Hodi FS, Friedlander P, Corless CL, et al. Major response to imatinib mesylate in KIT-mutated melanoma. J Clin Oncol. 2008;26:2046-51.

57. Quintás-Cardama A, Lazar AJ, Woodman SE, et al. Complete response of stage IV anal mucosal melanoma expressing KIT
Val560Asp to the multikinase inhibitor sorafenib. Nat Clin Pract Oncol. 2008;5:737-40.

58. Kim KB, Alrwas A. Treatment of KIT-mutated metastatic mucosal melanoma. Chin Clin Oncol. 2014;3:35.

59. Harbour JW, Roberson ED, Anbunathan H, et al. Recurrent mutations at codon 625 of the splicing factor SF3B1 in uveal melanoma. Nat Genet. 2013;45:133-5.

60. Kong Y, Krauthammer M, Halaban R. Rare SF3B1 R625 mutations in cutaneous melanoma. Melanoma Res. 2014;24:332-4.

61. Kong Y, Si L, Li Y, et al. Analysis of mTOR Gene aberrations in melanoma patients and evaluation of their sensitivity to PI3KAKT-mTOR pathway inhibitors. Clin Cancer Res. 2016;22:1018-27.

62. Villar VH, Nguyen TL, Terés S, et al. Escaping mTOR inhibition for cancer therapy: tumor suppressor functions of mTOR. Mol Cell Oncol. 2017;4:e1297284.

63. Aydin IT, Melamed RD, Adams SJ, et al. FBXW7 mutations in melanoma and a new therapeutic paradigm. J Natl Cancer Inst. 2014;106:dju107.

64. Jardim DL, Wheler JJ, Hess K, et al. FBXW7 mutations in patients with advanced cancers: clinical and molecular characteristics and outcomes with mTOR inhibitors. PLOS ONE. 2014;9: e89388.

65. Ma M, Dai J, Xu T, et al. Analysis of TSC1 mutation spectrum in mucosal melanoma. J Cancer Res Clin Oncol. 2018;144: 257-67.

66. Alexandrov LB, Nik-Zainal S, Wedge DC, et al. Signatures of mutational processes in human cancer. Nature. 2013;500:415-21.

67. Zhao T, Kong FW, Wang $\mathrm{H}$, et al. A long-term survivor with esophageal melanoma and pulmonary metastasis after single-stage esophagectomy and lobectomy: case report and literature review. Medicine (Baltimore). 2017;96:e7003. 